\title{
Robotic Label Applicator: Design, Development and Visual Servoing Based Control
}

\author{
Chyi-Yeu Lin, Asheber T. Wagshum and Trung-Son Le \\ Department of Mechanical Engineering, National Taiwan University of Science and Technology, Taipei, Taiwan
}

\begin{abstract}
Use of robotic arms and computer vision in manufacture, and assembly process are getting more interest as flexible customization is becoming priority over mass production as frontier industry practice. In this paper an innovative label applicator as end of arm tooling (EOAT) capable of dispensing and applying label stickers of various dimensions to a product is designed, fabricated and tested. The system incorporates a label dispenserapplicator and had eye-in-hand camera system, attached to 6-dof robot arm can autonomously apply a label sticker to the target position on a randomly placed product. Employing multiple advantages from different knowledge basis, mechanism design and vision based automatic control, offers this system distinctive efficiency as well as flexibility to change in manufacturing and assembly process with time and cost saving.
\end{abstract}

\section{Introduction}

Advance in manufacturing and assembly line methods are made regularly as new and more efficient ways of achieving the goal of increased efficiency are exposed. The equipment used and arrangement of theirs plays a great role for overall productivity of the line. New applications in mature and emerging industries, in addition to growing interest in automation pose pressure to insure innovation of flexible technologies and systems that can accommodate the changes. This in turn further expands the market for robotized manufacture and assembly [1].

Advance in manufacturing and assembly line methods are made regularly as new and more efficient ways of achieving the goal of increased efficiency are exposed. The equipment used and arrangement of theirs plays a great role for overall productivity of the line. New applications in mature and emerging industries, in addition to growing interest in automation pose pressure to insure innovation of flexible technologies and systems that can accommodate the changes. This in turn further expands the market for robotized manufacture and assembly [1].

Automation may range from employing a simple ordinary sensor to sophisticated industrial robot and machineries. Its effectiveness and value is measured in its ability to provide increased productivity for the user [2]. The robotic label applicator proposed in this paper can lie within the range of automation class. The raising sales of industrial robots each year, signifies robotics have high number of presence and taking central role in manufacturing industries as dependable work force. In fact, it is the dominant automation means in selected industries such as automotive, electrical/electronic equipment's, transportation, chemical products, etc. and this high population of robots in industry floors encourages its use and exploitation of its flexibility potential for customized automations.

Manufacturers are putting a considerable amount of effort to distinguish themselves by employing efficient automation and optimum equipment in their factory floor to remain competitive in local and international markets[3, 4]. Robotic arms are in high number in industries thus adding more sound value to their end of arm tooling (EOAT) will significantly improve productivity. The more flexible and smarter EOAT that actually acts with intelligence rather than depending on the arm itself is emerging in high numbers on robotic arm assisted assembly. As a result, we are starting to see new category of end effectors; alike of the mechanism presented in this paper, and manufacturers, and customers are highly tapping from this development.

Over decades, there has been progressive effort to incorporate computer vision and industrial robotics applications. Most common tasks are inspection, part handling and assembling. One early comprehensive review can be found in [5] in which classic vision algorithms using connectivity analysis, size/shape, position/orientation descriptors were briefly collected and explained. These early algorithms try to simplify $3 \mathrm{D}$ pose estimation problem to $2 \mathrm{D}$ case by taking advantage of conveyor belt picking or small part placement error. A more recent review shows persisting researches to solve general $3 \mathrm{D}$ vision problem by introducing more sophisticated sensory systems including stereo cameras, combining mono-camera and range sensor, structuredlight and mono-camera. In these systems, the cost for hardware and software development significantly increases. Regardless of quite a number of methods proposed, general part picking is still an unsolved challenge.

The vision algorithm used in this work has been a well-known problem in literature and was addressed by different terminologies. The early day literatures presented a bigger branch of research about structure and motion recovery $[6,7]$, where ours approach focuses on 
second, motion recovery subtopic. More recent literatures, Szeliski [8] describes a parametric feature-based alignment of two images in motion recovery with 6-DOF problems. It is well known that there is an ambiguity in translation solution by a scale factor [9, 10]. Zhang et al. proposed a solution for planar pattern [11] which required knowledge about the pattern's measurements as modelbased approach. Our approach is a $2 \mathrm{D}$ motion recovery from parametric feature-based image alignment. This takes advantage of our current application, which is used on planar objects, and the depth of vision can be fixed for simplicity. This results in a linear relation between image space and task space mapping 2D motion between these two spaces.

The rest of this paper is organized as follows: Section II focuses on conceptual explanation on the mechanism employed and the proposed hardware mechanism designed. Section III will present system integration and vision based control of the label applicator EOAT. In Section IV, experimentation and result will be discussed and lastly conclusion is presented in Section $\mathrm{V}$.

\section{Mechanism design}

In today's industries the demand and importance for product labeling and tagging is significantly high. This task is mostly done through a dedicated workstation, which is made for specific size, shape and position. Thus, when there is a need to change a product size and shape it necessitates a change in work flow setup and even the machineries mix and arrangements. Moreover manual labor is still used in some other industries. In this work a robotic mechanism for product labelling process presented, an intelligent product labeling and tagging.

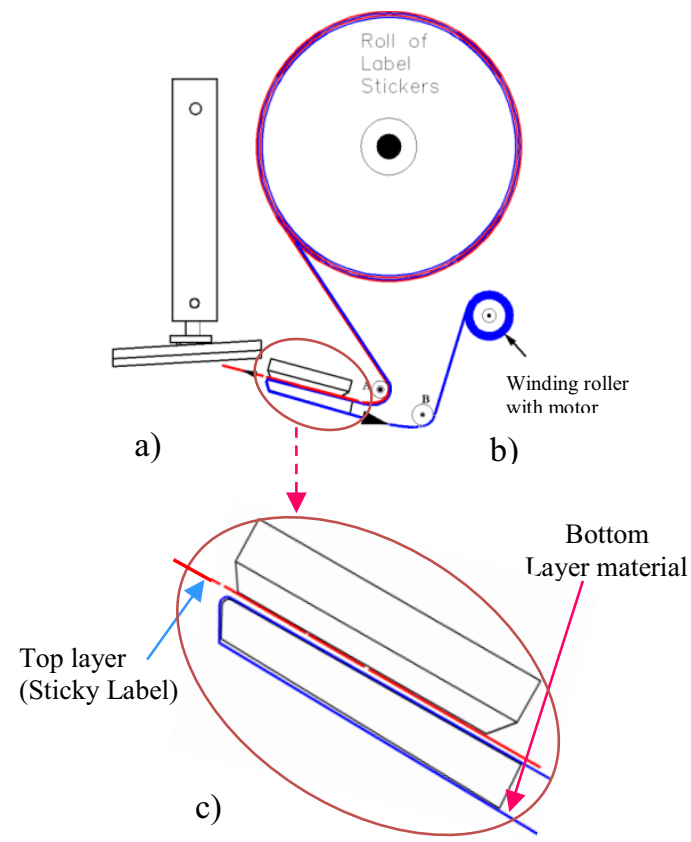

Figure 1.Schematic of sticker applicator working principle a) gripper unit b) label sticker delivery unit c) label peeler bed

\subsection{Conceptual design}

Product labeling in manufacturing processes or retail service have paramount importance and in many cases it is becoming crucial component. Usually this tagging or labeling is done through applying sticker on product surface or permanently engraved on the product body.

The two major steps, label supply and applying process, combined into one unit to form the operation principle of the robotic label applicator machine designed, manufactured and tested in this work, as schematically show in Figure 1. Thus label applicator system is modularly designed consisting of two basic subassemblies:

a) Label applicator module (Figure 1-a)and

b) Label supply module (Figure 1-b)

The label sticker is prepared in roll form, which is placed on passive roller on main frame of the system is made ready and supplied to applicator unit to paste to target position. The strip of the sticker label, label and underneath layer material, laid and pass over wedge shaped base (Figure 1-c magnified view) and other tensioning and guiding roller distributed along the path to deliver the sticker. The pull force applied by the winding roller (pull on under layer, blue strip, Figure 1b), powered by geared DC motor, will separate the upper sticky part (the label sticker, red strip, Figure 1b) from the underneath non sticky material. Once the sticker is ready the vacuum gripper is activated, signaled by the proximity sensor, to grab the label to apply to a target position. At each bend of the rollers, both driven or idlers, the contact between roller and strip of sticker paper must be insured for proper feeding without excessive slip [12]. Therefor the tension at the entry and exist of the roller will be different due to contact friction.

The distribution of idler and unwinding roller should consider avoidance of trough and wrinkle in the web and keep proper tension along web length [13]. However, the web length is relatively short such that most of this problem may not be a source of trouble in our design. The effect of roller speed, mass and consequently the force it induce can be neglected for the application considered in this work. Static analysis shows that tensions at entry and exit to non-driven roller (idlers A and B, Figure 1) can approximately relate as:

$$
\frac{T_{0}}{T_{i}}=e^{\mu \beta}
$$

Where $\mu$ is the coefficient of friction between roller surface stickers underneath player material and $\beta$ is wrap angle with roller. Thus equation (1) is needs to be taken into consideration while selecting the actuator and controller tuning.

\subsection{Mechanical design}

Labels or tags on products have multiple of purpose and effects for the producer and consumers. Automotive, medical device, food and beverage, pharmaceutical, 
personal care, computer and consumer electronics are among predominant industries where automated assembly and product labeling is highly employed and continuously improved. In current changing demand of consumer, the product life cycles are not long enough to support customized machine tools. This condition then encourages robust automated solution, design of innovative robotic label applicator mechanism. The applicator presented here is coupled to robot arm as end of arm tooling (EOAT) equipment. Thus, the robot arm will autonomously seek for the target position to apply the label sticker guided by visual servoing control technique with vision camera fitted on this applicator as feedback source.

The main contribution of this paper lies in the innovative design development and implementation of robotic labeling mechanism that supply the label and paste to target surface. The basic features of the applicator allow flexibility in manufacturing and assembly process due to its self-contained compact autonomous features. Applying label sticker anywhere on the object can be done easy without a need for reconfiguration of the system.

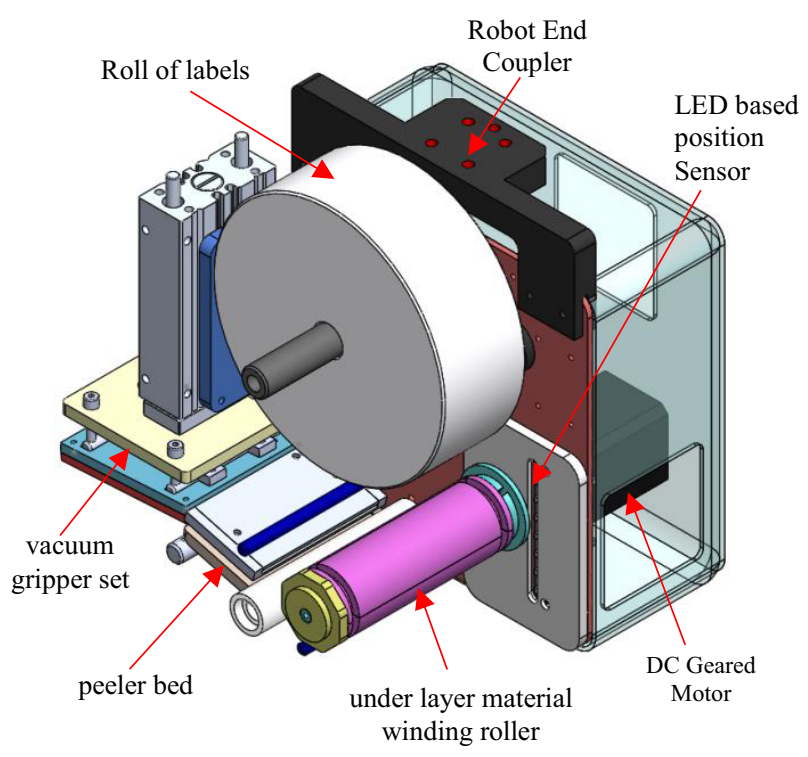

Figure 2. Label Applicator CAD model

To keep it light weight, the applicator most parts are designed for manufacture using rapid prototyping technology (3D printing) without losing structural strength required for its operation. As stated in the conceptual part the rotation of label dispensing roller, powered via DC geared motor, will separate the label from the underneath non sticky layer and makes it ready for the vacuum gripper. Table 1 shows the basic components of this robotic applicator.

The actuation unit includes a DC motor (output power $3.1 \mathrm{~W}$ ) with gearhead (total reduction ratio, 48:1), pneumatic piston and vacuum generator as can be seen in Figure 2. The controller regulates the DC motor to insure constant rate of label supply, which is achieved by feedback from line of LED array monitoring the diametrical size of underneath material collected by the winding motor.

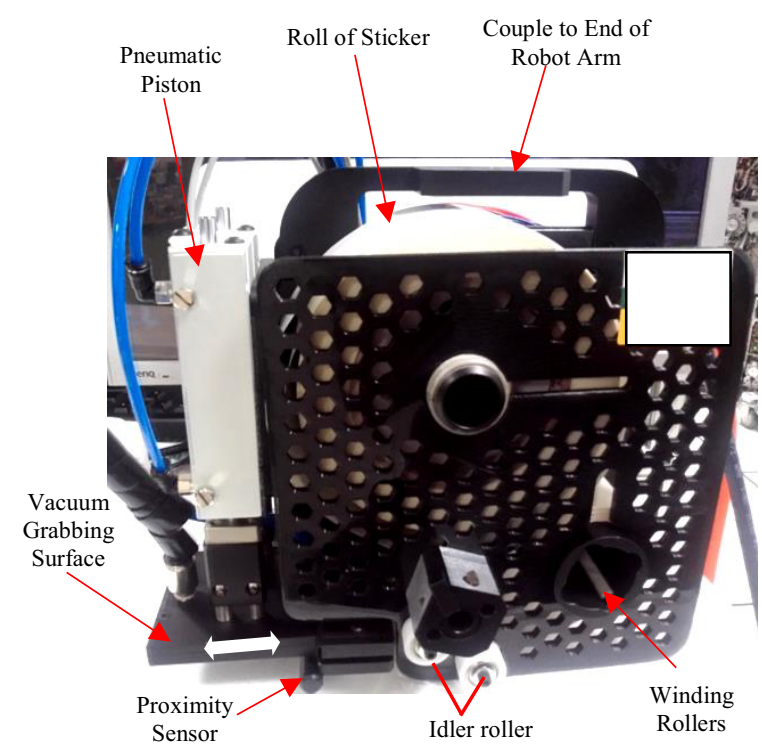

Figure 3. Complete Assembly of Label Applicator Prototype

Once grabbing surface hold the sticker it needs to move in the direction away from the peeler wedge edge to avoid interference while traveling down to the target area. The white thick arrow in Figure 3 shows the direction that sort of movement once the label is grabbed the grabbing surface moves $3 \mathrm{~mm}$ in the direction of the arrow to avoid collision with peeler bed.

Table 1. Applicator basic components.

\begin{tabular}{lc}
\hline \multicolumn{1}{c}{ Part Name } & Description \\
\hline Pneumatic gripper & $\begin{array}{l}\text { Gripe surface + set of vacuum } \\
\text { supply unit } \\
\text { Acrylic }\end{array}$ \\
Label rollers & Acrylic \\
DC Geared motor & IG32R 01004 \\
Solenoid Valves & Standard Set \\
Vision Camera & Raspberry Pi Camera \\
Robot arm end coupler & Aluminium \\
Micro -Controller & Raspberry pi 2 model B V1.1 \\
Proximity sensor & Standard \\
\hline
\end{tabular}

\section{System integration and control}

The embedded position, torque and proximity sensors (proximity sensor, position sensor) are shown in Figure $2 \& 3$. In typical control system diagram shown in Figure 4 the motor speed is controlled based on a feedback from line of proximity sensor LED distributes along the 
winding roller diameter monitoring the collected underneath layer material by the roller. Therefore, a constant speed of label deliver is achieved throughout the labeling process by controlling the speed of the driving motor based on this sensor feedback collected from the TMS32012810 based microprocessor.

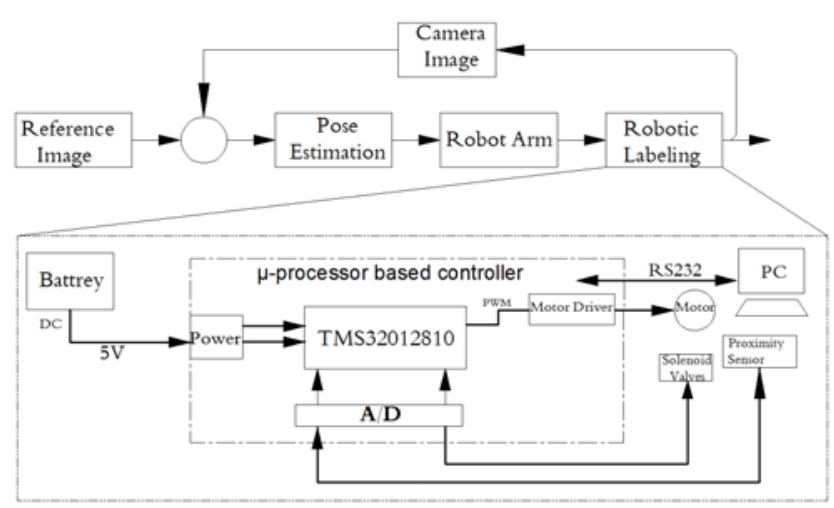

Figure 4. Control System Diagram

The operation of the system can be distinguished between the operation of the dispenser-applicator mechanism and of the vision-robot system. Therefore, the control involves two levels of scheme; the higher control stages where robot arm servoing is performed and labeling machine control in a second level (Figure 4). Raspberry Pi2 model Bv1.1 (BCM2836 ARM7 processor) was utilized as the main controller in the labelling machine and vision system control.

\subsection{Vision System}

A typical labeling task requires a target object to be placed at a known position and orientation, usually planar surface allowing a certain small error by an operator. This working condition provides a simplified assumption for vision and control processing. In particular, the placement error is restricted to a $2 \mathrm{D}$ motion and the known planar surface provides a fixed-depth pose estimation problem. Figure 5 shows the cooperation in control between main controller and vision module.

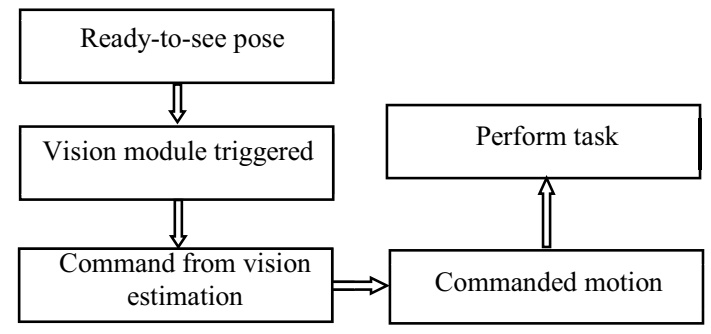

Figure 5. Vision based control operational flow

To perform the motion estimation in step 3 , the algorithm starts with feature point detection which uses good feature to track [14]. This is followed by a matching using optical flow and a robust registration with Euclidean parametric model to reject outliers (Figure 6).

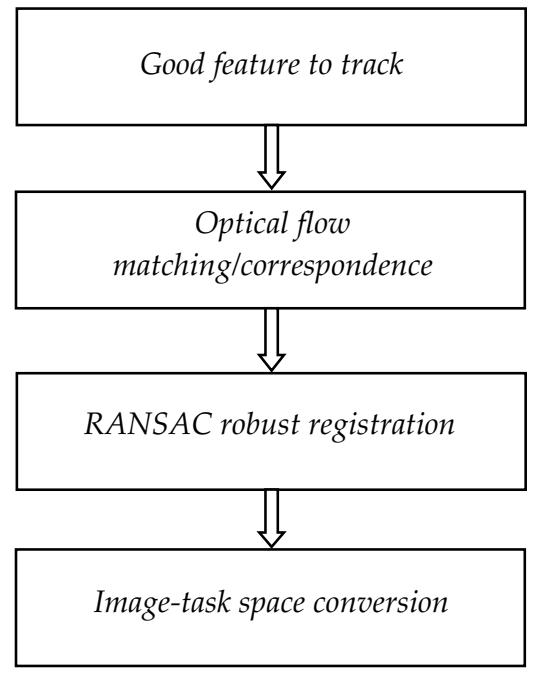

Figure 6. Motion estimation algorithm

The result motion solved in image-space is mapped to task space based on a linear relation $I=\alpha T$ where I, T are image-space and task-space measurements respectively; $\alpha$ is conversion scalar multi-disciplinary.

\subsection{Dispenser-applicator Mechanism}

The vision system operates according to Figure 5. At third step, the vision algorithm estimates the $2 \mathrm{D}$ motion to ready-for-tasking pose. After step5, straight-down motion, the controller triggers labeling process. It operates a dispensing motor and applicator gripper sequentially. The pneumatic gripper is fixed to a main frame on vertical guide, and moved to grasping direction simultaneously due to proximity sensor triggers the solenoid valves consequently the piston. Based on the information from the sensor the vacuum grasping surface on the pneumatic gripper will move to the bottom edge of the label sticker such that it sucks the sticker fully to its surface without damage. External compressed air supplied is distributed through the solenoid valves as depicted in Figure 7 to the two pneumatic pistons and vacuum grabbing surface.

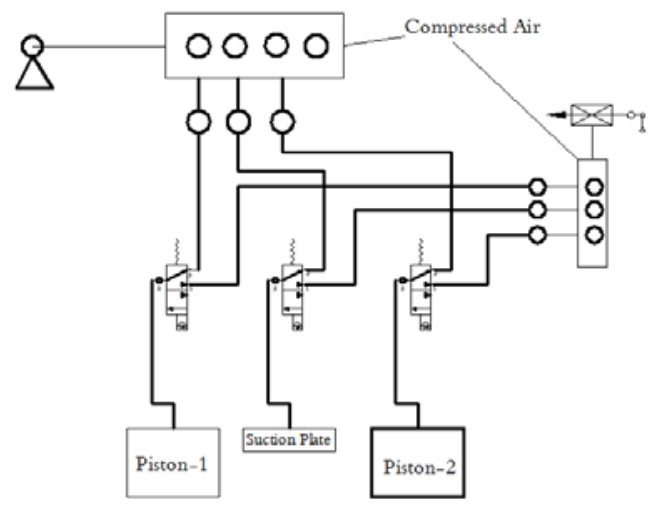

Figure 7. Schematics of the pneumatic circuits 


\section{Experiment and result}

To validate the performance of the robotic label applicator system proposed in this paper prototype is developed and experiment has been conducted. Figure 8 show the experimental set up to test the performance of the applicator machine. It is attached to end of $6 \mathrm{DOF}$ robot arm with own separate controller. Vision processing is accomplished on Raspberry PI embedded computer via captured images from PI camera. Vision module is setup to communicate with PC using TCP/IP protocol.

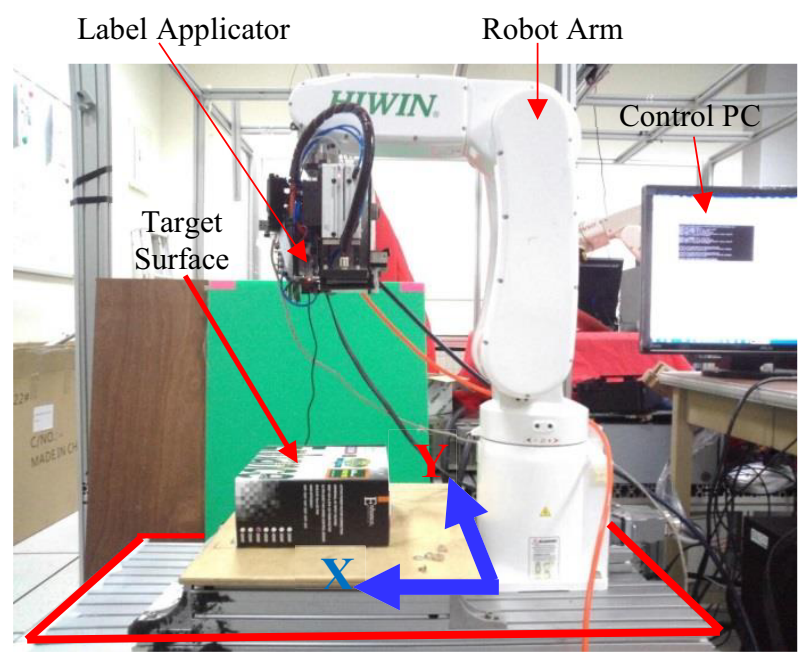

Figure 8. Experimental setup

The A4 sized chessboard template used in this test is shown in Figure 9a which is replaced for natural template in real-scenario targets due to the fact that some objects do not have feature points to be applied with current vision algorithm. The designed template provides the advantages for pose estimation of target pattern and the applied label by using straight-forward computer vision techniques as well as key-point features for vision module's algorithm. These pictures as can be seen from these captured images in Figure 9 b\&c.

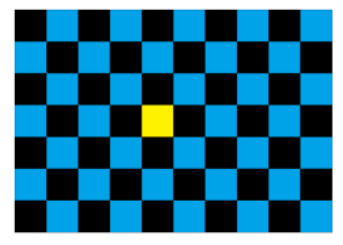

a)

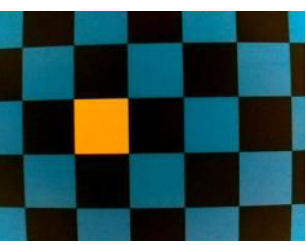

b)

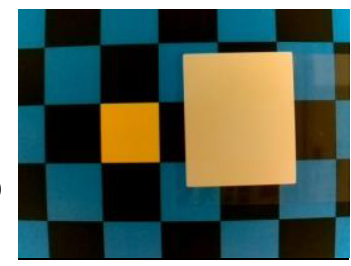

Figure 9. Template and reference pattern, reference pattern with label captured from PI camera

Experimental data collected for 30 repetitions shows some performance evaluation of the system (Figure10).
The overall control maintains a standard deviation of $\mathrm{dT}_{\mathrm{x}}=-0.386, \mathrm{dT}_{\mathrm{y}}=0.818, \mathrm{~d} \theta=0.5$ and mean value of $10.445,-6.325,0.5$ for case object is placed at $\mathrm{T}_{\mathrm{X}}=25$ $(\mathrm{mm}), \mathrm{T}_{\mathrm{y}}=5(\mathrm{~mm}), \theta=3^{0}$ deviated from reference position. Due to camera's field of view and vision algorithm, the object can be placed within an overall working range of $\mathrm{T}_{X}=30 \mathrm{~mm}, \mathrm{~T}_{\mathrm{y}}=30 \mathrm{~mm}$, and $\theta=15^{\circ}$.
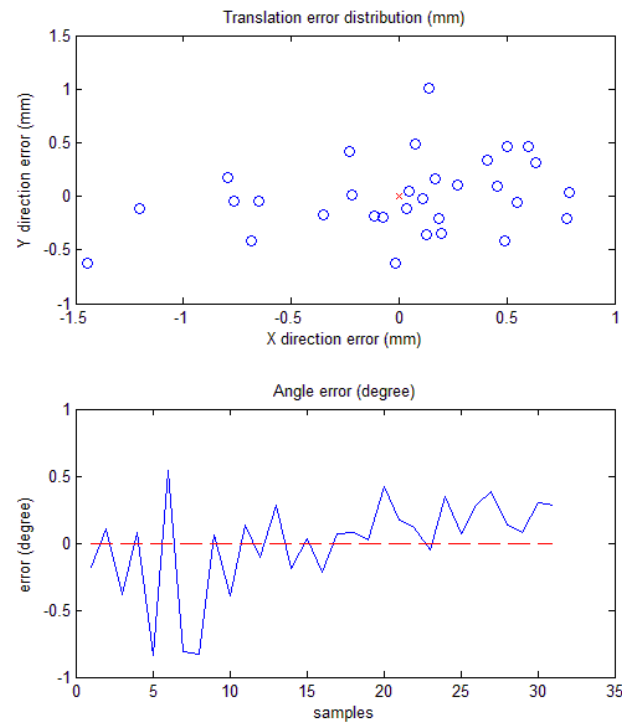

Figure 10. Performance error verification

Processing time of the system is also recorded. The graph shows 10 times of recording (Figure 10). Each record includes duration for vision processing, robot servoing and their total. Overall means are $1.7 \mathrm{~s}, 2.27 \mathrm{~s}$ and $3.98 \mathrm{~s}$ for vision processing, robot servoing and total processing respectively. Among these records, instance 1, 7 and 10 show overshoots of processing time. This is the result of sampling-based algorithm where the correct solutions are not returned in first little iteration for these instances.

\section{Conclusions}

In this paper an innovative new robotic label stickers or tags dispensing and pasting system is reported. Intuitive and more natural steps involved in design of this mechanism therefore it is less complex and highly flexible to adapt changes in system arrangement or label size and type change. From systematic point of view, the paper proposes a robotic solution with functionality, structure, actuation, and sensor configuration and control strategies considered carefully. Successful experimentation on the system demonstrates that it's fairly efficient and accurate in applying a label sticker on randomly oriented object with certain features on its surface. Its contribution for wide range of industries is enormous in terms of time and cost saving in addition to its easily customizable to a change in manufacturing lines. The current robotic mechanism designed is tasted on flat surface however, with minor change on grabbing surface; one can makes it suitable for curved surface as well. 


\section{REFERENCES}

1. C. M. P. Santos and M. J. Ferreira, "Control of an industrial desktop robot using computer vision and fuzzy rules," in Industrial Electronics, 2005. ISIE 2005. Proceedings of the IEEE International Symposium on, 2005, pp. 1297-1302 vol. 3.

2 F. Jovane, Y. Koren, and C. R. Boër, "Present and .Future of Flexible Automation: Towards New Paradigms," CIRP Annals - Manufacturing Technology, vol. 52, pp. 543-560, // 2003.

3. A. Fast-Berglund, T. Fässberg, F. Hellman, A. Davidsson, and J. Stahre, "Relations between complexity, quality and cognitive automation in mixed-model assembly," Journal of Manufacturing Systems, vol. 32, pp. 449-455, 7// 2013.

4. A. G. Abad, K. Paynabar, and J. J. Jin, "Modeling and Analysis of Operator Effects on Process Quality and Throughput in Mixed Model Assembly Systems," Journal of Manufacturing Science and Engineering, vol. 133, pp. 021016-021016, 2011.

5. G. J. Agin, "Computer Vision Systems for Industrial Inspection and Assembly," Computer, vol. 13, pp. 11-20, 1980.

6. O. Faugeras, N. Deriche, and N. Navab, "From optical flow of lines to 3D motion and structure," in Mustererkennung 1989. vol. 219, H. Burkhardt, K. Höhne, and B. Neumann, Eds., ed: Springer Berlin Heidelberg, 1989, pp. 522-530.

7. G. Toscani and R. Deriche, "Cooperation between 3D Motion Estimation and Token Trackers," in Multisensor Fusion for Computer Vision. vol. 99, J. K. Aggarwal, Ed., ed: Springer Berlin Heidelberg, 1993, pp. 153-153.
8. R. Szeliski, Computer Vision: Algorithms and Applications, 2011 ed.: Springer, 2011.

9. P. Corke, Robotics, Vision and Control: Fundamental Algorithms in MATLAB vol. 2011, 2011.

10. N. Navab, R. Deriche, and O. D. Faugeras, "Recovering 3D motion and structure from stereo and 2D token tracking cooperation," in Computer Vision, 1990. Proceedings, Third International Conference on, 1990, pp. 513-516.

11. Z. Zhang, "A Flexible New Technique for Camera Calibration," IEEE Trans. Pattern Anal. Mach. Intell., vol. 22, pp. 1330-1334, 2000.

12. H. Thanhtam, S. Hyeunhun, and L. Sangyoon, "Fuzzy control of the lateral position of a moving web in roll-to-roll processes," in Robotics and Biomimetics, 2008. ROBIO 2008. IEEE International Conference on, 2009, pp. 1637-1642.

13. S. Kee-Hyun and K. Soon-Oh, "The effect of tension on the lateral dynamics and control of a moving web," in Industry Applications Conference, 2005. Fourtieth IAS Annual Meeting. Conference Record of the 2005, 2005, pp. 529-536 Vol. 1.

14. J. Shi and C. Tomasi, "Good features to track," in Computer Vision and Pattern Recognition, 1994. Proceedings CVPR '94., 1994 IEEE Computer Society Conference on, 1994, pp. 593-600. 